\title{
Modeling of Financial Data: Comparison of the Truncated Lévy Flight and the $\operatorname{ARCH}(1)$ and $\operatorname{GARCH}(1,1)$ processes
}

\author{
Rosario N. Mantegna ${ }^{1}$ and H. Eugene Stanley ${ }^{2}$ \\ ${ }^{1}$ Istituto Nazionale per la Fisica della Materia, Unità di Palermo and Dipartimento di Energetica \\ ed Applicazioni di Fisica, Università di Palermo, Palermo, I-90128, ITALIA \\ ${ }^{2}$ Center for Polymer Studies and Dept. of Physics, Boston University, Boston, MA 02215 USA
}

\begin{abstract}
We compare our results on empirical analysis of financial data with simulations of two stochastic models of the dynamics of stock market prices. The two models are (i) the truncated Lévy flight recently introduced by us and (ii) the $\operatorname{ARCH}(1)$ and $\operatorname{GARCH}(1,1)$ processes. We find that the TLF well describes the scaling and its breakdown observed in empirical data, while it is not able to properly describe the fluctuations of volatility empirically detected. The $\operatorname{ARCH}(1)$ and $\operatorname{GARCH}(1,1)$ models are able to describe the probability density function of price changes at a given time horizon, but both fail to describe the scaling properties of the PDFs for short time horizons.
\end{abstract}




\section{INTRODUCTION}

Inspired by pioneering works on the analysis and modeling of economic and financial systems [1-3], a growing number of physicists are becoming involved in the analysis and modeling of financial markets [4-19]. In this lecture we consider the price dynamics of a stock index traded in a financial market. The most accepted paradigm in finance is that no arbitrage is present in financial markets i.e. there is no way to extract money from the market continuously and without risk [20].

In this lecture, we firstly recall results obtained by us [9, [1], by performing an empirical analysis of high-frequency data of one of the most important indices of the New York Stock Exchange, the Standard \& Poor's 500 (S\&P500) index. The results obtained in the empirical analysis are used as benchmarks for two stochastic processes used to model price dynamics in financial markets. The first model is the truncated Lévy flight (TLF), recently introduced by us [21]. The second stochastic process belongs to the class of autoregressive conditional heteroscedasticity $(\mathrm{ARCH})$ models [22] and to its generalization (GARCH) [23]. We address strengths and weaknesses of all three models in describing real financial data. We focus

our attention on the probability density functions (PDFs) of price changes at different time horizons, on the scaling properties of the PDFs and on the degree of stationarity of index changes.

\section{EMPIRICAL ANALYSIS}

We performed empirical analyses of the dynamics of indices of stock prices traded in financial markets [4,9, 11]. Our empirical analysis [9, 11] of the S\&P500 Index of the New York Stock Exchange shows that a non-Gaussian scaling of the PDF of price changes is present at short times (from $\Delta t=1$ to $\Delta t=1000$ trading minutes) while a breakdown from the non-Gaussian scaling is present for long times $(\Delta t>>1000$ trading minutes) [9, 11]. We performed our analysis by analyzing high frequency data recorded during the 6-year period 
1/84-12/89 (time intervals between successive records as short as 15 seconds are present in the data base). In our analysis [9], we define the trading time as a continuous time starting from the opening of the day until the closing, and then continuing with the opening of the next trading day. From this data base, we select the complete set of non-overlapping records separated by a time interval $\Delta t \pm \epsilon \Delta t$ (where $\epsilon$ is the tolerance, always less than 0.035 ). We denote the value of the $\mathrm{S} \& \mathrm{P} 500$ as $y(t)$, and we define $z(t) \equiv y(t)-y(t-\Delta t)$. In our analysis, we determine [9] the probability distribution $P(z)$ of index variations for different values of $\Delta t$. We select $\Delta t$ values that are logarithmically equally spaced ranging from 1 to 1000 min. The number of data in each set is decreasing from the maximum value of $493,545(\Delta t=1 \mathrm{~min})$ to the minimum value of $562(\Delta t=1000 \mathrm{~min})$. We note [9] that the distributions are non-Gaussian, indeed, they have wings larger than expected for a normal process.

We study the "probability of return to the origin" $P(z=0)$ as a function of $\Delta t$. With this choice, we are investigating the point of each probability distribution that is least affected by the noise introduced by the finiteness of the experimental data set. Our investigation of $P(0)$ versus $\Delta t$ in a log-log plot [9] shows that the data are well-fit by a straight line characterized by the slope $-0.712 \pm 0.025$. We observe a non-normal scaling behavior (slope $\neq-0.5)$ in an interval of trading time ranging from 1 to $1000 \mathrm{~min}$.

For short time horizons (from $\Delta t=1$ to $\Delta t=1000$ minutes), this empirical finding agrees with the model of a Lévy flight proposed by Mandelbrot in 1963 to model cotton price dynamics [1] or with the model of a Lévy walk [24]. In fact, if the central region of the distribution is well-described by a Lévy stable symmetrical distribution [25,

$$
L_{\alpha}(z, \Delta t) \equiv \frac{1}{\pi} \int_{0}^{\infty} \exp \left(-\gamma \Delta t q^{\alpha}\right) \cos (q z) d q,
$$

of index $\alpha$ and scale factor $\gamma$ at $\Delta t=1$, then the probability of return is given by

$$
P(0) \equiv L_{\alpha}(0, \Delta t)=\frac{\Gamma(1 / \alpha)}{\pi \alpha(\gamma \Delta t)^{1 / \alpha}} .
$$

By using the value -0.712 from the analysis of the probability of return, we obtain the index $\alpha=1.40 \pm 0.05$ [9]. 
We also check if the scaling extends over the entire probability distribution as well as $z=0$. All the distributions (with $\Delta t=1$ to $\Delta t=1000$ minutes) agree well with a Lévy stable distribution [9,26]. The distributions obtained with the highest temporal resolution $(\Delta t<10)$ show that in addition to the good agreement with the Lévy (non-Gaussian) profile observed for almost three orders of magnitude, an approximately exponential fall-off is present. The clear deviation of the tails of the distribution from the Lévy profile shows that the experimental tails are less fat than expected for a Lévy distribution.

The Lévy distribution has an infinite second moment (if $\alpha<2$ ) 225]. However, our empirical finding of an exponential (or stretched exponential) fall-off implies that the second moment is finite. This conclusion might at first sight seem to contradict our observation of Lévy scaling of the central part of the price difference distribution over fully three orders of magnitude. However, the contradiction is more apparent than real since, for example, the above findings are consistent with the theoretical predictions of the truncated Lévy flight 21].

\section{THE TRUNCATED LÉVY FLIGHT}

The truncated Lévy flight (TLF) has been introduced by Mantegna and Stanley in Ref. [21]. A TLF is defined as a stochastic process $\{x\}$ characterized by the following probability density function

$$
T(x) \equiv \begin{cases}0 & x>\ell \\ c_{1} L(x) & -\ell \leq x \leq \ell \\ 0 & x<-\ell\end{cases}
$$

where $L(x)$ is the symmetrical Lévy stable distribution of index $\alpha(0<\alpha \leq 2)$ and scale factor $\gamma(\gamma>0), c_{1}$ is a normalizing constant and $\ell$ is the cutoff length. In the following theoretical considerations, for the sake of simplicity, we set $\gamma=1$.

The central limit theorem (CLT) is fundamental to statistical mechanics. It states that when $n \rightarrow \infty$, the sum 


$$
z_{n} \equiv \sum_{i=1}^{n} x_{i}
$$

of $n$ stochastic variables $\{x\}$ that are statistically independent, identically distributed and with a finite variance, converges to a normal (Gaussian) stochastic process. Generally, $n \approx 10$ is sufficient to ensure convergence. In a dynamical system, Eq. (四) defines a random walk if the variable $x$ is the jump size performed after a time interval $\Delta t$ and $n$ is the number of time intervals. Here, the "number of variables" $n$ and the "time" $t=n \Delta t$ can be interchanged everywhere.

For low values of $n, P\left(z_{n}=0\right)$ takes a value very close to the one expected for a Lévy stable process

$$
P\left(z_{n}=0\right) \simeq L\left(z_{n}=0\right)=\frac{\Gamma(1 / \alpha)}{\pi \alpha n^{1 / \alpha}}
$$

For large values of $n, P\left(z_{n}=0\right)$ assumes the value predicted for a normal process,

$$
P\left(z_{n}=0\right) \simeq N\left(z_{n}=0\right)=\frac{1}{\sqrt{2 \pi} \sigma_{o}(\alpha, \ell) n^{1 / 2}}
$$

where $\sigma_{o}(\alpha, \ell)$ is the standard deviation of the TLF stochastic process $\{x\}$.

In the interval $1 \leq \alpha<2$, the crossover between the two regimes has been determined in Ref. 21] as:

$$
n_{\times} \approx A \ell^{\alpha}
$$

where $A$ is a function of $\alpha$ (the explicit form is given in Ref. [21]). The description of the convergence process does not depend crucially on the exact shape of the cut-off [27] and some results of Ref. [21] have been confirmed analytically for an exponential cut-off in Ref. [28].

By performing numerical simulations, we verified [21] that the probability of return to the origin indicates with high accuracy the degree of convergence of the process to one of the two asymptotic regimes.

The TLF model explains the empirical observations of (i) non-Gaussian scaling of the PDFs of price changes for short times; (ii) Lévy shape of the central part of the price 
change distributions for $\Delta t \leq 1000$ trading minutes; (iii) gradual convergence to a Gaussian process for long time horizons $(\Delta t>>1000$ trading minutes). However, not all the features observed in the S\&P 500 dynamics are described by the TLF model. The simplest version of the model cannot describe the short time memory (of the order of 20 minutes or less) observed in the empirical data [1],26] and also does not explain the empirical observation of the time dependence of the parameter $\gamma$ which is fluctuating with burst of activity localized in specific months [9,26]. The $\gamma$ parameter is related to what is called "volatility" in the economic literature 29].

\section{ARCH PROCESS}

ARCH stochastic models were introduced by Engle in 1982 [22]. They are stochastic models with autoregressive conditional heteroscedasticity, namely zero mean, uncorrelated stochastic processes with nonconstant variances conditional on the past. These models have a very interesting property: they might be locally unstationary (for short time intervals) but globally stationary for well defined ranges of the values of the control parameters. They are widely known in the economic literature [30], but they are almost unknown to the physics community in spite of the fact that they might also be useful in the description of physical problems.

The simplest $\mathrm{ARCH}$ model is the $\mathrm{ARCH}(1)$ model defined as a random variable $Z$ which is characterized at time $t$ by a variance $\sigma_{t}^{2}$ given by

$$
\sigma_{t}^{2}=\alpha_{0}+\alpha_{1} Z_{t-1}^{2}
$$

where $Z_{t-1}$ is a random variable selected from a set of random variables characterized by a Gaussian distribution with zero mean and standar deviation $\sigma_{t-1} \cdot \alpha_{0}$ and $\alpha_{1}$ are the control parameters of the stochastic process.

The most general ARCH stochastic process, the ARCH(n) process is defined by

$$
\sigma_{t}^{2}=\alpha_{0}+\alpha_{1} Z_{t-1}^{2}+\ldots \ldots \ldots \ldots+\alpha_{n} Z_{t-n}^{2}
$$


where $\alpha_{0}, \ldots, \alpha_{n}$ are control parameters and $Z_{t-1}, \ldots, Z_{t-n}$ are random variables drawn from sets of random variables with Gaussian distributions of zero mean and standard deviations $\sigma_{t-1}, \ldots, \sigma_{t-n}$ respectively. In spite of the fact that $\sigma_{t}$ is showing an intermittent-like behavior, the overall process $\{Z\}$ is stationary on a long time scale for a wide range of the control parameters. For example, it has been proven by Engle that the $\mathrm{ARCH}(1)$ process has finite variance for $\alpha_{1}<1$, and finite fourth moment for $3 \alpha_{1}^{2}<1$ [22].

We simulate several $\mathrm{ARCH}(1)$ process to investigate the dynamics of the unconditional probability density function $P\left(Z_{n \Delta t}\right)$ at different time horizons $n \Delta t\left(Z_{n \Delta t} \equiv \sum_{i=1}^{n} Z_{t-i}\right)$. For each simulation we also study the scaling properties of the "probability of return to the origin" $P\left(Z_{n \Delta t}=0\right)$ as a function of $n \Delta t$. We select the values of the control parameters to investigate $\mathrm{ARCH}(1)$ processes which are characterized by the same unconditional variance observed in our empirical investigation of the S\&P500 dynamics (namely $\sigma^{2}=2.57 \cdot 10^{-3}$ ) and by different values of the kurtosis $\kappa$ of the $\{Z\}$ process. For an $\mathrm{ARCH}(1)$ process the unconditional variance is given by 22]

$$
\sigma^{2}=\frac{\alpha_{0}}{1-\alpha_{1}}
$$

while the kurtosis is [22]

$$
\kappa=\frac{3\left(1-\alpha_{1}^{2}\right)}{1-3 \alpha_{1}^{2}}
$$

We focus our attention on three cases:

(i) $\alpha_{0}=0.00231$ and $\alpha_{1}=0.1$. In this case $\sigma^{2}=2.57 \cdot 10^{-3}$ and $\kappa=3.06$. The value of $\kappa$ is very close to the one expected for a Gaussian stochastic process $(\kappa=3)$;

(ii) $\alpha_{0}=0.00112$ and $\alpha_{1}=0.564$. With these values of the control parameters the variance and the kurtosis are $\sigma^{2}=2.57 \cdot 10^{-3}$ and $\kappa=43$. This value of $\kappa$ is approximately the same value observed in the empirical analysis of the S\&P500 changes for time intervals $\Delta t=1$ minute;

(iii) $\alpha_{0}=0.00109$ and $\alpha_{1}=0.575$. Values of the control parameters implying the same variance as above but a very high value for the kurtosis $(\kappa=247)$. 
By varying the values of the control parameters, it is of course possible to make the shape of the PDF $P(Z)$ more leptokurtic than a Gaussian distribution. The presence of a given degree of leptokurtosis does not imply directly scaling properties of $P\left(Z_{n \Delta t}\right)$ PDFs strongly different from the Gaussian scaling. By studying the "probability of return to the origin", we find that an approximate scaling behavior is present in $\mathrm{ARCH}(1)$ stochastic processes for short times $(n \Delta t \leq 100)$. We find that the values of the scaling exponent best describing the above-cited time evolution of $P\left(Z_{n \Delta t}=0\right)$ are 2.02, 1.93 and 1.85 respectively. These values are very close to the scaling exponent 2 observed for a Gaussian stochastic process. Hence an $\mathrm{ARCH}(1)$ process is not able to describe the scaling properties empirically observed in the stochastic dynamics of the S\&P500 for $\Delta t<1000$ minutes (where the scaling exponent is 1.4$)$.

\section{GARCH PROCESS}

$\mathrm{ARCH}(1)$ model is the simplest autoregressive model. In the following, we will consider a less simple autoregressive model, the $\operatorname{GARCH}(1,1)$ model. The $\operatorname{GARCH}(1,1)$ model is widely studied in the economic literature [30]. In 1986 generalized ARCH or GARCH(p,q) models were proposed [23]. These models are more flexible than ARCH models in the lag structure. They are defined by the relation

$$
\sigma_{t}^{2}=\alpha_{0}+\alpha_{1} Z_{t-1}^{2}+\ldots \ldots+\alpha_{p} Z_{t-p}^{2}+\beta_{1} \sigma_{t-1}^{2}+\ldots \ldots+\beta_{q} \sigma_{t-q}^{2}
$$

where the constants $\alpha_{0}, \ldots, \alpha_{p}, \beta_{1}, \ldots, \beta_{q}$ are the control parameters of the GARCH stochastic process. The simplest GARCH process, the $\operatorname{GARCH}(1,1)$, is often studied in the modeling of prices of financial assets. $\operatorname{GARCH}(1,1)$ processes are unconditional stationary with finite variance and fourth moment if $1-\alpha_{1}-\beta_{1}>0$ and $1-\beta_{1}^{2}-2 \alpha_{1} \beta_{1}-3 \alpha_{1}^{2}>0$ respectively. Empirical analyses of stock market price data have shown (see for example [31]) that a good choice of the parameter $\beta_{1}$ is $\beta_{1}=0.9$. Accordingly, we set $\beta_{1}=0.9$ in our simulations and we set the remaining control parameters $\alpha_{0}$ and $\alpha_{1}$ to the values $\alpha_{0}=2.3 \quad 10^{-5}$ and $\alpha_{1}=0.09105$. With this choice of control parameters, the unconditional variance [32 


$$
\sigma^{2}=\frac{\alpha_{0}}{1-\alpha_{1}-\beta_{1}}
$$

of the process $\{Z\}$ is approximately equal to the value observed in the S\&P500 data. The kurtosis 32

$$
\kappa=\frac{6 \alpha_{1}^{2}}{1-\beta_{1}^{2}-2 \alpha_{1} \beta_{1}-3 \alpha_{1}^{2}}+3
$$

also assumes the value measured in the empirical analysis of the S\&P500 data $(\kappa=43)$. The simulated $\operatorname{GARCH}(1,1)$ process has an unconditional $\operatorname{PDF} P\left(Z_{\Delta t}\right)$ which mimics very well the one observed in the S\&P500 data with a time interval $\Delta t=1$ minute [9]. We also study the probability of return to the origin to determine if scaling is observed for the PDFs of this process. We observe a scaling behavior for a wide range of time $(n \Delta t<10,000)$. The measured scaling exponent is 1.88, a value close to the Gaussian scaling exponent and rather different from the scaling exponent found in empirical data $(\alpha=1.4)$.

In summary, the $\mathrm{GARCH}(1,1)$ process fails to properly describe the scaling properties of the S\&P500 index detected for $\Delta t<1000$ minutes. However, $\operatorname{GARCH}(1,1)$ is able to give an accurate description of the $\Delta t=1$ minute PDF using as control paremeters $\beta_{1}=0.9$ and

obtaining the values of $\alpha_{0}$ and $\alpha_{1}$ from the values of $\sigma^{2}$ and $\kappa$ measured from the empirical data.

\section{DISCUSSION}

Our study shows that the problem of the complete stochastic characterization of index (or price) dynamics in a financial market is an open question. For example, both models considered here have strengths and limitations, and modifications of them are needed to reach a more satisfactory agreement with the results of empirical analyses. The problem of stochastic modeling of price dynamics comprises fundamental and applied aspects. The fundamental aspects are related to the theoretical modeling of a nonlinear complex system evolving without known conservation laws in the presence of quenched and external noise. The applied aspects are related to the role that the exact shape of the PDF of stock returns 
and the time evolution of the variance of stock returns (volatility in the economic literature [29]), plays in the pricing of derivative financial products [33. An extremely important activity performed everyday in financial markets.

We think that the mixing of empirical analyses, modeling, simulations and comparison between empirical data and simulations constitutes a scientific procedure that will allow us to eventually find the most accurate and "parsimonious" stochastic model describing index (or price) dynamics.

\section{ACKNOWLEDGMENTS}

We thank INFM, MURST and NSF for financial support. 


\section{REFERENCES}

[1] B. B. Mandelbrot, J. Business 36 (1963) 394.

[2] L. P. Kadanoff, Simulation 16 (1971) 261.

[3] E. W. Montroll and W. W. Badger, Introduction to Quantitative Aspects of Social Phenomena (Gordon and Breach, New York, New York, 1974).

[4] R. N. Mantegna, Physica A 179 (1991) 232.

[5] W. Li, International Journal of Bifurcation and Chaos 1 (1991) 583.

[6] H. Takayasu, H. Miura, T. Hirabayashi, and K. Hamada, Physica A184 (1992) 127.

[7] P. Bak, K. Chen, J. A. Scheinkman, and M. Woodford, Ricerche Economiche 47 (1993) 3.

[8] J.-P. Bouchaud and D. Sornette, J. Phys. I France 4 (1994) 863.

[9] R. N. Mantegna and H. E. Stanley, Nature 376 (1995) 46.

[10] S. Ghashghaie, W. Breymann, J. Peinke, P. Talkner and Y. Dodge, Nature 381 (1996) 767.

[11] R. N. Mantegna and H. E. Stanley Nature 383 (1996) 587; R. N. Mantegna and H. E. Stanley, in [Proc. International Conference on Pattern Formation in Fluids and Materials], Physica A 239, 255-266 (1997).

[12] A. Arneodo, J. P. Bouchaud, R. Cont, J. F. Muzy, M. Potters, D. Sornette, "Comment on Turbulent Cascades in Foreign Exchange Markets" (preprint);

[13] M. Levy and S. Solomon, Int. J. Mod. Phys. C7 (1996) 595.

[14] S. Galluccio, G. Caldarelli, M. Marsili and Y.-C. Zhang, Physica A 245 (1997) 423.

[15] P. Bak, M. Paczuski, and M. Shubik, Physica A 246 (1997) 430. 
[16] M. Potters, R. Cont, J.-P. Bouchaud, "Financial markets as adaptative systems" (preprint, 1996)

[17] N. Vandewalle and M. Ausloos, Physica A 246 (1997) 454.

[18] Y. Liu, P. Cizeau, M. Meyer, C.-K. Peng, and H. E. Stanley, Physica 245 (1997) 437.

[19] P. Cizeau, Y. Liu, M. Meyer, C.-K. Peng, and H. E. Stanley, Physica 245 (1997) 441.

[20] J. E. Ingersoll, Theory of Financial Decision Making (Rowman \& Littlefield, Savage, Maryland, 1987).

[21] R. N. Mantegna and H. E. Stanley, Phys. Rev. Lett. 73 (1994) 2946; R. N. Mantegna and H. E. Stanley, in Lévy Flights and Related Topics in Physics [PROC. 1994 INTERNATIONAL CONF. ON LÉVY FLIGHTS], edited by M. F. Shlesinger, G. M. Zaslavsky, and U. Frisch (Springer, Berlin, 1995), pp. 300-312.

[22] R. F. Engle, Econometrica 50 (1982) 987.

[23] T. Bollerslev, J. Econometrics 31 (1986) 307.

[24] M. F. Shlesinger, G. M. Zaslavsky and J. Klafter, Nature 363 (1993) 31.

[25] P. Lévy, Théorie de l'Addition des Variables Aléatoires (Gauthier-Villars, Paris, 1937).

[26] R. N. Mantegna and H. E. Stanley, in Proceedings of the International Summer School of Physics "E. Fermi", The Physics of Complex Systems, edited by Mallamace F. and Stanley H. E. (IOS Press, Amsterdam, 1997) pp 473.

[27] M. F. Shlesinger, Phys. Rev. Lett 74 (1995) 4959.

[28] I. Koponen, Phys. Rev. E52 (1995) 1197.

[29] G. W. Schwert, The Journal of Finance 44 (1989) 1115.

[30] T. Bollerslev, R. Y. Chou, and K. F. Kroner, J. Econometrics 52 (1992) 5. 
[31] V. Akgiray, J. Business 62 (1989) 55.

[32] R. T. Baillie and T. Bollerslev,J. Econometrics 52 (1992) 91.

[33] J. C. Hull, Options, Futures, and Other Derivatives, Third Edition (Prentice Hall, Upper Saddle River, NJ, 1997). 